\title{
A discretised nonlinear eigenvalue problem with many spurious branches of solutions
}

\author{
C.A. Stuart and G.Vuillaume \\ Département de Mathématiques, \\ Ecole Polytechnique Fédérale de Lausanne, \\ CH-1015 Lausanne, \\ Switzerland \\ e-mail: charles.stuart@epfl.ch \\ e-mail: gregory.vuillaume@epfl.ch
}

\begin{abstract}
We treat an example of a nonlinear eigenvalue problem in $L^{2}(0,1)$ which can be solved explicitly. It has a single branch of nontrivial solutions. Discretisation reduces the problem to a finite dimensional one having many branches of nontrivial solutions. We investigate the convergence of these approximate solutions.
\end{abstract}

\section{Introduction}

In this paper we consider an example in the theory of equations of the type

$$
S u-N(u)=\lambda u \quad \text { for } u \in H \backslash\{0\}
$$

where $S: H \rightarrow H$ is a positive self-adjoint operator acting on a real Hilbert space $H$ and $N: H \rightarrow H$ is a non-linear operator with $N(0)=0$. Our goal is to expose, through a particular example of (1), a problem which can occur when we consider finite dimensional approximations of (1). In fact this example shows us that the approximation has many branches of solutions which have no relevence for the initial problem (1). We show moreover why these branches of solutions do not converge to solutions of the initial problem.

In Section 2 we introduce our basic example of equation (1). Then, in Section 3, we make a discretisation to reduce the initial problem to a finite dimensional problem. In Sections 4 and 5 we show that only one branch of solutions of the approximate problem converges to a branch of solutions of the initial problem and that the solutions on the other branches do not converge to solutions of the initial problem. Finally, in Section 6 , we consider the same problem as above in relation to the spectrum of $S$. 
General results concerning the existence and bifurcation of solutions for (1) are given in [1-4] and variants of the example discussed below are used to illustrate the sharpness of the conclusions in [1] and [4]. Our observations concerning discrete approximations apply to such variants too but we feel it is sufficient to exhibit them in the simplest context. More general situations are treated in [5].

\section{Example}

Let $H$ be the real Hilbert space $L^{2}(0,1)$ with the usual scalar product $<.$, . $>$ and norm $\|$.$\| . A bounded, positive, self-adjoint operator, S: H \rightarrow H$, is defined by

$$
S u=f u \quad \text { for } u \in H
$$

where $f:[0,1] \rightarrow \mathbb{R}$ is the function defined by $f(x)=x^{2}$. If $\sigma(S)$ denotes the spectrum of $S$ (and $\rho(S)=\mathbb{R} \backslash \sigma(S)$ denotes the resolvent set of $S$ ), it is easy to check that

$$
\sigma(S)=\overline{\operatorname{Imf}}=[0,1]
$$

and that $S$ has no eigenvalues. For $\sigma>0$, we define $N: H \rightarrow H$ by

$$
N(u)(x)=|I(u)|^{\sigma} I(u) \quad \text { for } x \in[0,1]
$$

where

$$
I(u)=\int_{0}^{1} u(x) d x
$$

Since $|I(u)| \leq\|u\|$, it follows that $N(u) \in H$. With the operators defined by (2) and (3), equation (1) becomes

$$
x^{2} u(x)-|I(u)|^{\sigma} I(u)=\lambda u(x) \quad \text { a.e. on }[0,1] \text { for }(\lambda, u) \in \mathbb{R} \times[H \backslash\{0\}]
$$

and as we now show, it can be solved explicitly.

Since $I(u)$ is constant on $[0,1]$, any solution of (4) must have the form

$$
u(x)=c d_{\lambda}(x) \quad \text { a.e. on }[0,1]
$$

where $c \neq 0$ is a constant and $d_{\lambda}(x)=\left(x^{2}-\lambda\right)^{-1}$. Furthermore, a function satisfying (5) belongs to $H$ if and only if $\lambda \in \rho(S)$. Returning to (4), we find that $c$ must be chosen so that

$$
1=|c|^{\sigma}\left|I\left(d_{\lambda}\right)\right|^{\sigma} I\left(d_{\lambda}\right)
$$

which means that $\lambda \in \rho(S)$ must be chosen so that $I\left(d_{\lambda}\right)>0$. In this case, it is clear that

$$
\left\{\lambda \in \rho(S): I\left(d_{\lambda}\right)>0\right\}=(-\infty, 0) .
$$


Let

$$
\mathcal{C}=\left\{\left(\lambda, \pm u_{\lambda}\right): \lambda \in(-\infty, 0)\right\}
$$

where $u_{\lambda}(x)=I\left(d_{\lambda}\right)^{-(\sigma+1) / \sigma} \cdot d_{\lambda}(x)$ a.e. on $[0,1]$. It follows from $(6)$ that

$$
\{(\lambda, u) \in \mathbb{R} \times H \backslash\{0\}:(4) \text { is satisfied }\}=\mathcal{C} .
$$

From (7) we see that the only possible bifurcation point for (4) is 0 . To determine whether or not 0 is a bifurcation point we must study lim $\left\|u_{\lambda}\right\|$ as $\lambda \rightarrow 0_{-}$. We have

$$
\begin{aligned}
\left\|u_{\lambda}\right\|= & \left\{\int_{0}^{1} \frac{1}{x^{2}-\lambda} d x\right\}^{-(\sigma+1) / \sigma} \cdot\left\{\int_{0}^{1} \frac{1}{\left(x^{2}-\lambda\right)^{2}} d x\right\}^{1 / 2} \\
= & \left\{\frac{|\lambda|^{1 / \sigma}}{2(1+|\lambda|) \arctan (1 / \sqrt{|\lambda|})^{2(\sigma+1) / \sigma}}\right. \\
& \left.+\frac{|\lambda|^{(2-\sigma) / 2 \sigma}}{2 \arctan (1 / \sqrt{|\lambda|}))^{2(\sigma+1) / \sigma-1}}\right\}^{1 / 2},
\end{aligned}
$$

from which it follows that

$$
\lim _{\lambda \rightarrow 0_{-}}\left\|u_{\lambda}\right\|=0 \Longleftrightarrow \sigma<2 .
$$

Thus 0 is a bifurcation point of (4) if and only if $\sigma<2$. The different bifurcation diagrams for equation (4) with different values of $\sigma$ are shown in Figure 1.

\section{Approximation of the example}

Let $n \in \mathbb{N}^{*}=\{1,2, \ldots\}$. We define $f_{n}:[0,1] \rightarrow \mathbb{R}$ by

$$
f_{n}(x)= \begin{cases}\left(\frac{k}{n}\right)^{2} & \text { if } \frac{k}{n} \leq x<\frac{k+1}{n}, k=0,1, \ldots, n-1, \\ \left(\frac{n-1}{n}\right)^{2} & \text { if } x=1 .\end{cases}
$$

Next we define a subspace of $H$ by

$$
Y_{n}=\left\{\varphi \in H: \varphi \text { is constant a.e. on }\left(\frac{k}{n}, \frac{k+1}{n}\right) \text { for } k=0,1, \ldots, n-1\right\} \text {. }
$$

Clearly $\operatorname{dim} Y_{n}=n$. We observe that $f_{n} \in Y_{n}$ and that $f_{n} \rightarrow f$ uniformly on $[0,1]$ as $n \rightarrow \infty$.

Now we can define the approximate problem in dimension $n$, or $n$ approximate problem, by

$$
S_{n} u-N(u)=\mu u \quad \text { with }(\mu, u) \in \mathbb{R} \times\left[Y_{n} \backslash\{0\}\right],
$$



(a) $\left\|u_{\lambda}\right\|$ with $\sigma=1 / 5$.
(b) $\left\|u_{\lambda}\right\|$ with $\sigma=1$.
(c) $\left\|u_{\lambda}\right\|$ with $\sigma=2$.
(d) $\left\|u_{\lambda}\right\|$ with $\sigma=6$.

Figure 1: $\left\|u_{\lambda}\right\|$ with different values of $\sigma$.

where $S_{n}: Y_{n} \rightarrow Y_{n}$ is the positive, self-adjoint operator defined by

$$
S_{n} \varphi=f_{n} \varphi \text { for } \varphi \in Y_{n}
$$

Clearly we have

$$
\sigma\left(S_{n}\right)=\overline{\operatorname{Imf_{n}}}=\left\{\left(\frac{k}{n}\right)^{2}: 0 \leq k \leq n-1\right\},
$$

each point $(k / n)^{2}$ is a simple eigenvalue of $S_{n}$ and the bifurcation points of (10) are all the elements of $\sigma\left(S_{n}\right)$. With the operator defined by (11), equation (10) becomes

$f_{n}(x) \varphi(x)-|I(\varphi)|^{\sigma} I(\varphi)=\mu \varphi(x) \quad$ a.e. on $[0,1]$ for $(\mu, \varphi) \in \mathbb{R} \times\left[Y_{n} \backslash\{0\}\right]$.

Once again it is possible to solve this equation explicitly. Following the same way as above, we find that $\mu$ must be chosen so that $\mu \in \rho\left(S_{n}\right)$ and 
$I\left(\left[f_{n}-\mu\right]^{-1}\right)>0$. We note that

$$
I\left(\left[f_{n}-\mu\right]^{-1}\right)=\frac{1}{n} \sum_{j=0}^{n-1}\left[\left(\frac{j}{n}\right)^{2}-\mu\right]^{-1}
$$

and so one can check that for each given $k=1, \ldots, n-1$ and for $\mu \in$ ]$\left(\frac{k-1}{n}\right)^{2},\left(\frac{k}{n}\right)^{2}\left[, I\left(\left[f_{n}-\mu\right]^{-1}\right)\right.$ increases from the limit $-\infty\left(\right.$ as $\left.\mu \rightarrow\left(\frac{k-1}{n}\right)_{+}^{2}\right)$ to $+\infty\left(\right.$ as $\left.\mu \rightarrow\left(\frac{k}{n}\right)_{-}^{2}\right)$. Therefore, for each $k=1, \ldots, n-1$, there exists $\left.\xi_{k}^{n} \in\right]\left(\frac{k-1}{n}\right)^{2},\left(\frac{k}{n}\right)^{2}[$ such that, for $\mu \in]\left(\frac{k-1}{n}\right)^{2},\left(\frac{k}{n}\right)^{2}[$, we have

$$
I\left(\left[f_{n}-\mu\right]^{-1}\right) \begin{cases}>0 & \text { if } \mu>\xi_{k}^{n} \\ =0 & \text { if } \mu=\xi_{k}^{n} \\ <0 & \text { if } \mu<\xi_{k}^{n} .\end{cases}
$$

Then we have

$$
\left\{\mu \in \rho\left(S_{n}\right): I\left(\left[f_{n}-\mu\right]^{-1}\right)>0\right\}=(-\infty, 0) \cup \cup_{k=1}^{n-1}\left(\xi_{k}^{n},\left(\frac{k}{n}\right)^{2}\right) .
$$

Let $J_{0}^{n}=(-\infty, 0)$ and $J_{k}^{n}=\left(\xi_{k}^{n},(k / n)^{2}\right)$ for $k=1,2, \ldots, n-1$. Then, for $\mu \in \cup_{k=0}^{n-1} J_{k}^{n}$, we set

$$
u_{\mu}^{n}(x)=I\left(\left[f_{n}-\mu\right]^{-1}\right)^{-(\sigma+1) / \sigma} \cdot\left(f_{n}(x)-\mu\right)^{-1}
$$

for $x \in[0,1]$. Then, for $k=0,1, \ldots, n-1$,

$$
\mathcal{C}_{k}^{n}=\left\{\left(\mu, \pm u_{\mu}^{n}\right): \mu \in J_{k}^{n}\right\}
$$

is the branch of solutions of the $n$-approximate problem (10) which bifurcates from the point $\left((k / n)^{2}, 0\right)$ in $\mathbb{R} \times Y_{n}$. Furthermore

$$
\cup_{k=0}^{n-1} \mathcal{C}_{k}^{n}=\left\{(\mu, \varphi) \in \mathbb{R} \times\left[Y_{n} \backslash\{0\}\right]:(12) \text { is satisfied }\right\} .
$$

Note that $\mu \rightarrow\left\|u_{\mu}^{n}\right\|$ is continuous on $J_{k}^{n}$ for $k=0,1, \ldots, n-1$. In fact,

$$
\left\|u_{\mu}^{n}\right\|=\frac{\left(\frac{1}{n} \sum_{j=0}^{n-1}\left[\left(\frac{j}{n}\right)^{2}-\mu\right]^{-2}\right)^{1 / 2}}{\left(\frac{1}{n} \sum_{j=0}^{n-1}\left[\left(\frac{j}{n}\right)^{2}-\mu\right]^{-1}\right)^{\frac{\sigma+1}{\sigma}}} .
$$

As $\mu$ approches $\xi_{k}^{n}$ from above, it is clear that $\left\|u_{\mu}^{n}\right\| \rightarrow \infty$ since the denominator tends to zero by the definition of $\xi_{k}^{n}$, whereas the numerator remains bounded away from zero.

As $\mu$ approches $\left(\frac{k}{n}\right)^{2}$ from below, we claim that $\left\|u_{\mu}^{n}\right\| \rightarrow 0$. To see this, let $t=\left(\frac{k}{n}\right)^{2}-\mu$ and observe that

$$
\left\|u_{\mu}^{n}\right\|=\frac{\left(\frac{1}{n} t^{-2}+\alpha(\mu)\right)^{1 / 2}}{\left(\frac{1}{n} t^{-1}+\beta(\mu)\right)^{\frac{\sigma+1}{\sigma}}}
$$


where

$$
\alpha(\mu)=\frac{1}{n} \sum_{j=0, j \neq k}^{n-1}\left[\left(\frac{j}{n}\right)^{2}-\mu\right]^{-2}
$$

and

$$
\beta(\mu)=\frac{1}{n} \sum_{j=0, j \neq k}^{n-1}\left[\left(\frac{j}{n}\right)^{2}-\mu\right]^{-1} .
$$

Thus

$$
\left\|u_{\mu}^{n}\right\|=\frac{\left(\frac{1}{n} t^{2 / \sigma}+t^{\frac{2(\sigma+1)}{\sigma}} \alpha(\mu)\right)^{1 / 2}}{\left(\frac{1}{n}+t \beta(\mu)\right)^{\frac{\sigma+1}{\sigma}}}
$$

where $t^{\frac{2(\sigma+1)}{\sigma}} \alpha(\mu) \rightarrow 0$ and $t \beta(\mu) \rightarrow 0$ as $t \rightarrow 0_{+}$. It follows that $\left\|u_{\mu}^{n}\right\| \rightarrow 0$ as $\mu$ approches $\left(\frac{k}{n}\right)^{2}$ from below.

Remark. If we solve equation (12) in the set $H$ instead of $Y_{n}$ we verify that the solutions belong to $Y_{n}$ and are exactly the same as those obtained by solving the $n$-approximate problem and the bifurcation points are also the same. Thus we can directly consider the $n$-approximate problem in $H$.

The bifurcation diagram of the $n$-approximate problem is drawn in Figure 2 .

Figure 2: Bifurcation diagram of $S_{n} u-N(u)=\mu u$.

Observing Figures 1 and 2 we want to show the two following results when $n \rightarrow+\infty$ :

1. The branches $\mathcal{C}_{0}^{n}$ bifurcating from $\mu=0$ converge to the branch $\mathcal{C}$ of exact solutions of (4) as $n \rightarrow \infty$. However $\mathcal{C}$ bifurcates from 0 if and only if $0<\sigma<2$.

2. Solutions on the other branches do not converge. 


\section{The first result}

We begin by studying the behaviour of the branches $\mathcal{C}_{0}^{n}$ as $n \rightarrow \infty$. We fix $\mu \in(-\infty, 0)$. By (14) we know that $u_{\mu}^{n}$ is a solution of (12) for all $n \geq 1$. We check that, in the notation of (7),

$$
\lim _{n \rightarrow \infty} u_{\mu}^{n}(x)=I\left(d_{\mu}\right)^{-(\sigma+1) / \sigma} \cdot d_{\mu}(x)=u_{\mu}(x) \quad \text { a.e. on }[0,1]
$$

and the convergence is uniform on $[0,1]$ since $\left(f_{n}-\mu\right)^{-1} \rightarrow d_{\mu}$ uniformly on $[0,1]$ and $I\left(\left[f_{n}-\mu\right]^{-1}\right) \rightarrow I\left(d_{\mu}\right)$. Thus $\left(\mu, u_{\mu}^{n}\right) \in \mathcal{C}_{0}^{n}$ converges to the solution $\left(\mu, u_{\mu}\right) \in \mathcal{C}$ of $(4)$ for all $\mu \in(-\infty, 0)$. Now we have already shown that

$$
\lim _{\mu \rightarrow 0_{-}}\left\|u_{\mu}\right\|=0 \Longleftrightarrow \sigma<2 .
$$

Note however that for each $n \in \mathbb{N}^{*},\left\|u_{\mu}^{n}\right\| \rightarrow 0$ as $\mu \rightarrow 0_{-}$for all $\sigma>0$. The Figure 3 show the form of $\mathcal{C}_{0}^{n}$ for $n=5,10,15$ and $\sigma=1 / 5,1,2,6$ (in fact we represent in these figures $\left\{\left(\mu,\left\|u_{\mu}^{n}\right\|\right):\left(\mu, \pm u_{\mu}^{n}\right) \in \mathcal{C}_{0}^{n}\right\}$ and the thick curve represents $\left\{\left(\mu,\left\|u_{\mu}\right\|\right):\left(\mu, \pm u_{\mu}\right) \in \mathcal{C}\right\}$ for the respective $\left.\sigma\right)$.

(a)

(b)

(c)

(d)

Figure 3: $\mathcal{C}_{0}^{n}$ with $n=5,10,15$ and $\sigma=1 / 5$ (a), 1 (b), 2 (c), 6 (d). 


\section{The second result}

The second phenomenon we want to understand is why the other branches of solutions for the $n$-approximate problem do not converge to a branch of solutions for the initial problem as $n \rightarrow \infty$. To do that we consider the sequence of the $2 n$-approximate problems since the point $1 / 4=(n / 2 n)^{2}$ is the bifurcation point for all the branches $\mathcal{C}_{n}^{2 n}$ and we consider the limit when $n \rightarrow \infty$. It is clear that the following reasoning can be applied for any bifurcation point except 0 of an approximate problem of any dimension $n$.

For all $R>0$ we can define a sequence

$$
\left(\lambda_{n}, v_{n}\right) \in \mathcal{C}_{n}^{2 n} \text { and }\left\|v_{n}\right\|=R
$$

Thus we want to show that the limit of the branches of solutions does not exist when $n \rightarrow \infty$. To do this we show that $\left(v_{n}\right)_{n \geq 1}$ converges weakly to 0 when $n \rightarrow \infty$. By (13) we have

$v_{n}(x)=\left(\frac{1}{2 n} \sum_{k=0}^{2 n-1}\left[\left(\frac{k}{2 n}\right)^{2}-\lambda_{n}\right]^{-1}\right)^{-(\sigma+1) / \sigma} \cdot\left(f_{2 n}(x)-\lambda_{n}\right)^{-1}$ a.e. on $[0,1]$.

Lemma 5.1 For any $\delta \in(0,1 / 2)$ and any $R>0$, the sequence $\left\{\left(\lambda_{n}, v_{n}\right)\right\}$ defined by (16) has the properties that $\lambda_{n} \rightarrow 1 / 4$ and $v_{n}(x) \rightarrow 0$ uniformly on $\left[0, \frac{1}{2}-\delta\right] \cup\left[\frac{1}{2}+\delta, 1\right]$.

Proof: Let

$$
a_{n}=\frac{1}{2 n} \sum_{k=0}^{2 n-1}\left[\left(\frac{k}{2 n}\right)^{2}-\lambda_{n}\right]^{-2}
$$

and

$$
b_{n}=\frac{1}{2 n} \sum_{k=0}^{2 n-1}\left[\left(\frac{k}{2 n}\right)^{2}-\lambda_{n}\right]^{-1}
$$

Since $\lambda_{n} \in J_{n}^{2 n}$ we know that $b_{n}=I\left(\left[f_{2 n}-\lambda_{n}\right]^{-1}\right)>0$ and that $\lambda_{n} \in$ $\left(\left(\frac{n-1}{2 n}\right)^{2}, \frac{1}{4}\right)$. Hence $\lambda_{n} \rightarrow 1 / 4$.

Furthermore, from (17), it follows that

$$
\left\|v_{n}\right\|=\frac{a_{n}^{1 / 2}}{b_{n}^{(\sigma+1) / \sigma}}
$$

and so

$$
a_{n}^{1 / 2}=R \cdot b_{n}^{(\sigma+1) / \sigma} \quad \text { for all } n \in \mathbb{N}^{*} .
$$


Let us show that $a_{n} \rightarrow \infty$ as $n \rightarrow \infty$. In fact, for $0 \leq k \leq n-1$,

$$
\begin{aligned}
{\left[\left(\frac{k}{2 n}\right)^{2}-\lambda_{n}\right]^{2} } & \leq\left(\frac{k}{2 n}-\sqrt{\lambda_{n}}\right)^{2}\left(\frac{k}{2 n}+\sqrt{\lambda_{n}}\right)^{2} \\
& \leq\left(\sqrt{\lambda_{n}}-\frac{k}{2 n}\right)^{2}
\end{aligned}
$$

since $\frac{n-1}{2 n}<\sqrt{\lambda_{n}}<\frac{1}{2}$. But $\left(\sqrt{\lambda_{n}}-\frac{k}{2 n}\right)^{2} \leq\left(\frac{1}{2}-\frac{k}{2 n}\right) \frac{1}{2}$ for $0 \leq k \leq n-1$ and so

$$
\begin{aligned}
a_{n} & \geq \frac{1}{2 n} \sum_{k=0}^{n-1} \frac{4}{1-\frac{k}{n}}=2 \sum_{k=0}^{n-1} \frac{1}{n-k} \\
& =2 \sum_{i=1}^{n} \frac{1}{i} \rightarrow \infty \text { as } n \rightarrow \infty .
\end{aligned}
$$

From (18), we see that $b_{n}^{(\sigma+1) / \sigma} \rightarrow \infty$ as $n \rightarrow \infty$. For $\delta \in(0,1 / 2)$, there exists $n_{\delta}>0$ such that $\sqrt{\lambda_{n}} \in[1 / 2-\delta / 2,1 / 2)$ for all $n \geq n_{\delta}$ and so

$$
\begin{aligned}
\left|f(x)-\lambda_{n}\right| & =\left|x^{2}-\lambda_{n}\right|=\left|x-\sqrt{\lambda_{n}}\right|\left(x+\sqrt{\lambda_{n}}\right) \\
& \geq \frac{\delta}{2}\left(x+\sqrt{\lambda_{n}}\right) \geq \frac{\delta}{2}\left(\frac{1}{2}-\frac{\delta}{2}\right)=\frac{\delta(1-\delta)}{4}
\end{aligned}
$$

for all $x \in\left[0, \frac{1}{2}-\delta\right] \cup\left[\frac{1}{2}+\delta, 1\right]$.

Furthermore, since $f_{2 n} \rightarrow f$ uniformly on $[0,1]$, there exists $N(\delta) \geq n_{\delta}$ such that

$$
\left|f_{2 n}(x)-f(x)\right| \leq \frac{\delta(1-\delta)}{8} \text { for all } x \in[0,1] \text { and all } n \geq N(\delta) .
$$

Hence for $n \geq N(\delta)$ and $x \in\left[0, \frac{1}{2}-\delta\right] \cup\left[\frac{1}{2}+\delta, 1\right]$,

$$
\begin{aligned}
\frac{\delta(1-\delta)}{4} & \leq\left|f(x)-\lambda_{n}\right| \leq\left|f(x)-f_{2 n}(x)\right|+\left|f_{2 n}(x)-\lambda_{n}\right| \\
& \leq \frac{\delta(1-\delta)}{8}+\left|f_{2 n}(x)-\lambda_{n}\right|
\end{aligned}
$$

and consequently

$$
\left|f_{2 n}(x)-\lambda_{n}\right| \geq \frac{\delta(1-\delta)}{8} .
$$

Finally for $n \geq N(\delta)$ and $x \in\left[0, \frac{1}{2}-\delta\right] \cup\left[\frac{1}{2}+\delta, 1\right]$, we have shown that

$$
\left|v_{n}(x)\right| \leq b_{n}^{-(\sigma+1) / \sigma} \frac{8}{\delta(1-\delta)}=\frac{R}{\sqrt{a_{n}}} \frac{8}{\delta(1-\delta)}
$$

where $a_{n} \rightarrow \infty$ as $n \rightarrow \infty$. Thus $v_{n} \rightarrow 0$ uniformly on $\left[0, \frac{1}{2}-\delta\right] \cup\left[\frac{1}{2}+\delta, 1\right]$.

We can now show that $\left(v_{n}\right)_{n \geq 1}$ converges weakly to 0 which implies that $\left(v_{n}\right)_{n \geq 1}$ does not converge in $H$. 
Lemma 5.2 For any $R>0$, the sequence $\left(v_{n}\right)_{n \geq 1}$ defined by (16) converges weakly to 0 in $H$ but contains no strongly convergent subsequence.

Proof: Fix $\varphi \in H$ and $\epsilon>0$. To show that $v_{n} \rightarrow 0$ weakly in $H$, it is enough to show

$$
\limsup _{n \rightarrow \infty}\left|\left\langle v_{n}, \varphi\right\rangle\right| \leq \epsilon .
$$

For this we choose $\delta \in\left(0, \frac{1}{2}\right)$ small enough so that

$$
\int_{\frac{1}{2}-\delta}^{\frac{1}{2}+\delta} \varphi^{2}(x) d x \leq\left(\frac{\epsilon}{R}\right)^{2} .
$$

Then

$$
\begin{aligned}
\left|<v_{n}, \varphi>\right| \leq & \int_{|x-1 / 2| \leq \delta}\left|v_{n}(x)\right||\varphi(x)| d x \\
& +\max _{|x-1 / 2| \geq \delta}\left|v_{n}(x)\right| \int_{|x-1 / 2| \geq \delta}|\varphi(x)| d x \\
\leq & \left\{\int_{|x-1 / 2| \leq \delta} v_{n}^{2} d x\right\}^{1 / 2}\left\{\int_{|x-1 / 2| \leq \delta} \varphi^{2} d x\right\}^{1 / 2} \\
& +\max _{|x-1 / 2| \geq \delta}\left|v_{n}(x)\right| \int_{0}^{1}|\varphi| d x \\
\leq & R \cdot \frac{\epsilon}{R}+\|\varphi\| \max _{|x-1 / 2| \geq \delta}\left|v_{n}(x)\right|
\end{aligned}
$$

and Lemma 5.1 shows that $\lim \sup _{n \rightarrow \infty}\left|\left\langle v_{n}, \varphi\right\rangle\right| \leq \epsilon$ as required.

If $\left\{v_{n_{i}}\right\}$ is a subsequence of $\left\{v_{n}\right\}$ such that $\left\|v_{n_{i}}-v\right\| \rightarrow 0$ as $i \rightarrow \infty$ for some $v \in H$, then $v_{n_{i}} \rightarrow v$ as $i \rightarrow \infty$ and so $v=0$, showing that $\left\|v_{n_{i}}\right\| \rightarrow 0$ as $i \rightarrow \infty$. But $\left\|v_{n}\right\|=R>0$ for all $n \in \mathbb{N}^{*}$ and so there is no such subsequence.

Remark. With an analogous reasoning it is possible to extend these results to a function $f$ which is more general.

\section{The linear problem}

In this section we consider the linearization of (1)

$$
S u=\lambda u \quad \text { with }(\lambda, u) \in \mathbb{R} \times[H \backslash\{0\}] .
$$

Since $S$ has no eigenvalues it is clear that this equation has no solution $(\lambda, u)$ with $u \neq 0$. We also consider the $n$-approximate problem of (19)

$$
S_{n} u=\mu u \quad \text { with }(\mu, u) \in \mathbb{R} \times\left[Y_{n} \backslash\{0\}\right]
$$


where $S_{n}$ and $Y_{n}$ are defined as above. In this case $S_{n}$ has the $n$ following eigenvalues

$$
\left\{\mu_{k}^{n}:=\left(\frac{k}{n}\right)^{2}: 0 \leq k \leq n-1\right\} .
$$

Each eigenvalue is simple and the eigenspace of $\mu_{k}^{n}$ is

$$
E_{k}^{n}=\operatorname{span}\left\{X_{k}^{n}\right\}
$$

where $X_{k}^{n}$ is the characteristic function of $\left[\frac{k}{n}, \frac{k+1}{n}\right)$. The bifurcation diagram is then represented in Figure 4.

Figure 4: Bifurcation diagram of $S_{n} u=\mu u$.

Let us consider what happens to the branches of solutions as $n \rightarrow \infty$. To do that we consider once again the sequence of the $2 n$-approximate problems. As before, $1 / 4=\mu_{n}^{2 n}$ is an eigenvalue for all these problems. Once again we fix $R>0$ and we define a sequence $\left(v_{n}\right)_{n \geq 1}$ where $v_{n} \in E_{n}^{2 n}$ is such that $\left\|v_{n}\right\|=R$. It is easy to show that this sequence $\left(v_{n}\right)_{n \geq 1}$ converges weakly to 0 and thus does not contain a subsequence converging strongly in $H$. Finally let us observe that for the linear problem this reasoning is also valid for the point 0 .

\section{References}

[1] H.-P. Heinz and C.A.Stuart. Solvability of Nonlinear Equations in Spectral Gaps of the Linearization. Nonlinear Analysis, Theory, Methods and Applications, 19(2):145-165, 1992.

[2] H.-P. Heinz, T. KüPper, and C.A. Stuart. Existence and Bifurcation of Solutions for Nonlinear Perturbations of the Periodic Schrödinger Equation. Journal of Differential Equations, 100(2):341-354, December 1992. 
[3] T. Küpper and C.A. Stuart. Bifurcation into Gaps in the Essential Spectrum. Journal für die reine und angewandte Mathematik, 409:1-34, 1990.

[4] T. KÜPPER and C.A. Stuart. Necessary and Sufficient Conditions for Gap-Bifurcation. Nonlinear Analysis, Theory, Methods and Applications, 18(9):893-903, 1992.

[5] G. Vuillaume. Bifurcation du spectre essentiel: Théorie et exemples. Diploma's Work, Ecole Polytechnique Fédérale de Lausanne, 1999-2000. 